Ophthalmologe 2012 $\cdot 109: 317-318$

DOI 10.1007/s00347-012-2549-4

๑) Springer-Verlag 2012

\section{U. Schiefer}

Visuelle Funktionsdiagnostik und Neuro-Ophthalmologie, Department für Augenheilkunde, Universität Tübingen

\title{
Funktionelle Glaukomdiagnostik
}

\section{Ein essenzielles Element der visuellen Leistungs- und Verlaufsbeurteilung}

Die glaukomatöse Optikusneuropathie gehört zu den häufigsten Erblindungsursachen der westlichen Welt. Morphologische Kriterien und morphometrische Verfahren sind hierbei zwar wichtige objektive Elemente der Glaukom-(Früh-) Diagnostik und -Verlaufskontrolle. Entscheidende Grundlage für die Beurteilung der Leistungsfähigkeit bzw. -einschränkung, für Begutachtungsfragen und auch für die Eignungs- und Verlaufsbeurteilung ist und bleibt hingegen die visuelle Funktion: Immerhin nehmen wir mehr als 90\% unserer Umfeldinformationen über das visuelle System auf [1]! Ziel der hier zusammengestellten Beiträge ist es, einen aktuellen, kritischen Überblick über das breite Spektrum glaukombezogener funktionsdiagnostischer Verfahren zu liefern.

\section{1) Mehr als 90\% unserer Umfeldinformationen nehmen wir über das visuelle System auf}

Der Beitrag von Katja Göbel et al. eröffnet den Reigen dieser Arbeiten: Er gibt einen Überblick wesentlicher Ganglienzellsubtypen sowie deren Glaukomsuszeptibilität und stellt danach Funktionsprinzipien, Stärken und Schwächen verschiedener „Flimmer-Kontrasttests" (globale Flimmerschwellen, Flimmerperimetrie, Rauschfeldkampimetrie, Frequenzverdopplungsperimetrie und flimmerbasierte Gestaltperimetrie) vor.
Felix Tonagel et al. berichten in ihrem Beitrag über die verschiedenen konventionellen Lichtsinn-perimetrischen Verfahren, die zugehörigen Strategien sowie die Befundvisualisierung bzw. -auswertung. Ein „Highlight“ dieser Arbeit ist die tabellarische Zusammenstellung aktuell gebräuchlicher perimetrischer Progressionskriterien bekannter Glaukomstudien. Auf die Blau-Gelb-Perimetrie („short wavelength automated perimetry", SWAP; Übersicht in $[2,3]$ ) wird im Rahmen dieses Leitthemas nicht speziell eingegangen, weil es bis heute kein klinisch einsetzbares Verfahren gibt, das die für die Verlaufskontrolle essenzielle individuelle "Vergilbung" der menschlichen Augenlinse messen und adäquat berücksichtigen könnte. Alexander F. Scheuerle et al. analysieren in ihrem Beitrag, welche der vorgenannten konventionellen perimetrischen Methoden im Falle fortgeschrittener oder gar präterminaler Glaukomstadien anwendbar sind. Die Arbeit von Klaus Rohrschneider et al. beleuchtet kritisch den (begrenzten) Nutzen funduskontrollierter mikroperimetrischer Verfahren im Rahmen der funktionellen Glaukomdiagnostik.

Karolina Skorkovská et al. wenden sich in ihrem Beitrag der objektiven pupillenbezogenen Glaukomdiagnostik zu. Der Swinging-flashlight-Test erlaubt hierbei einen globalen, schnellen und - durch den Einsatz von Graufiltern sogar quantifizierbaren - Vergleich der visuellen Afferenz beider Augen. Ortsbezogene Aussa- gen liefert die Pupillenperimetrie/-kampimetrie mit sequenziellen Stimulusdarbietungen bzw. die multifokale Pupillographie mit wechselnden wabenförmigen Stimulationsmustern (M-Sequenzen). Der Beitrag geht abschließend auf eine erst vor wenigen Jahren genauer charakterisierte Gruppe retinaler neuronaler Elemente in Gestalt der sog. intrinsisch photosensitiven Melanopsin-haltigen retinalen Ganglienzellen (ipRGCs) ein.

Im letzten Beitrag dieser Serie befassen sich Charlotte M. Poloschek und Michael Bach mit den Möglichkeiten der elektrophysiologischen Glaukomdiagnostik. Nach Ihrer Einschätzung zeigen hier das Muster- (Pattern-)Elektroretinogramm (PERG) sowie eine Komponente des Ganzfeld-ERG („photopic negative response", PhNR) die erfolgversprechendsten Resultate.

Abschließend darf ich auch auf diesem Wege nochmals allen Mitgliedern des Autorenteams für deren Unterstützung und Engagement danken. Frau Sabrina Kohl vom Springer Verlag bin für ihre wertvolle Hilfe bei der Koordination dieser Beiträge sehr verbunden.

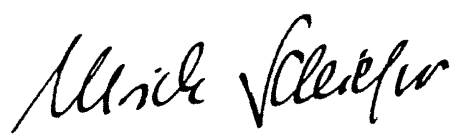

Ulrich Schiefer 


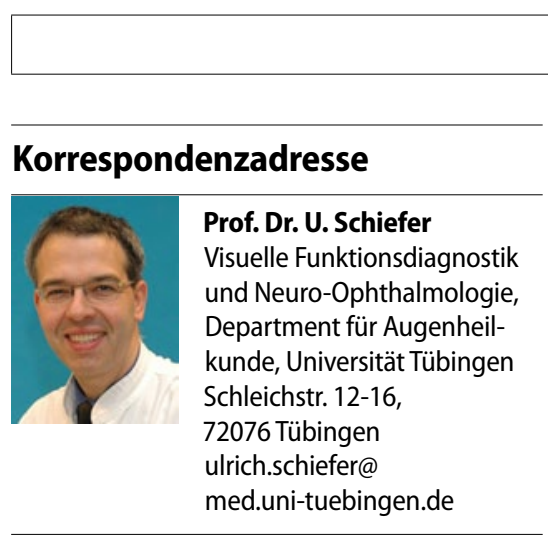

Interessenkonflikt. Der korrespondierende Autor weist auf folgende Beziehungen hin: Berater/Consultant der Firmen HAAG-STREIT, Schweiz; Berater/Consultant der Fa. SERVIER, Frankreich. Unterstützungen von nachfolgenden Firmen: ALCON, Deutschland; DAIMLER, Deutschland; MSD (CHIBRET), USA; PFIZER, USA; PHARM ALLERGAN, Deutschland und USA.

\section{Literatur}

1. Taylor JF (1982) Vision and driving. Practitioner 226:885-889

2. Fogagnolo P, Rossetti L, Ranno S et al (2008) Shortwavelength automated perimetry and frequencydoubling technology perimetry in glaucoma. Prog Brain Res 173:101-124

3. Racette L, Sample PA (2003) Short-wavelength automated perimetry. Ophthalmol Clin North Am 16:227-236

\section{Fachnachrichten}

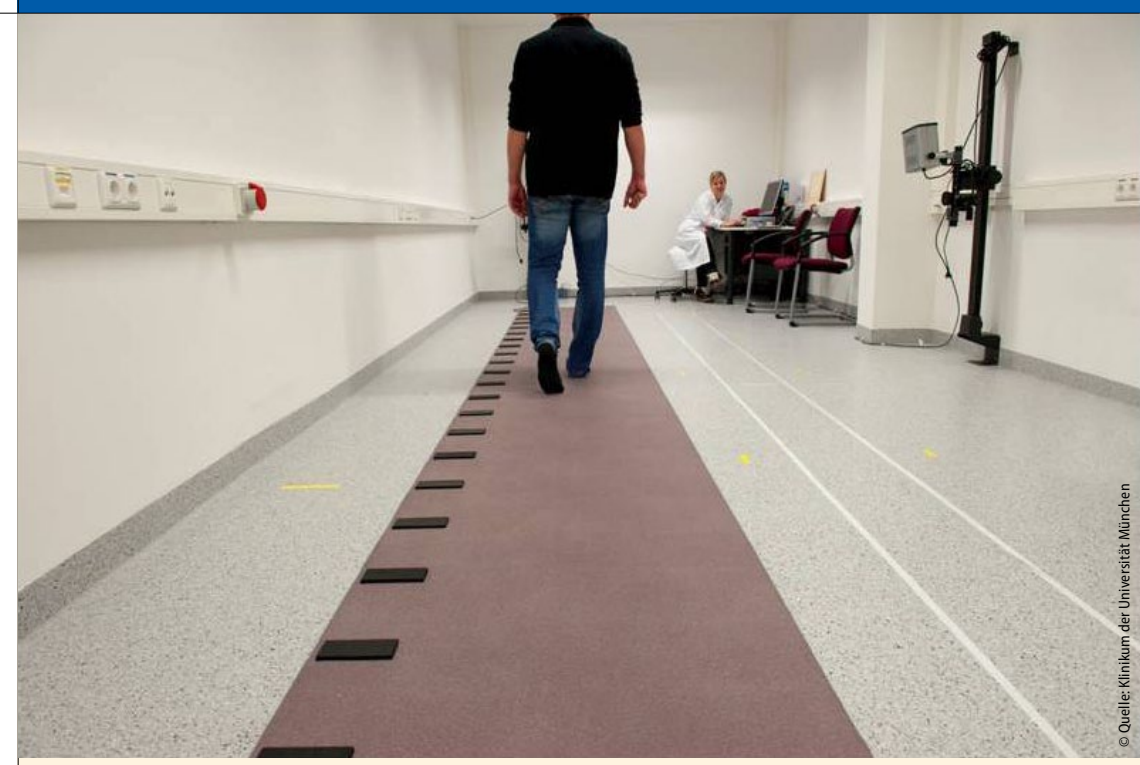

\section{VERTIGO XVI - Münchner Schwindel-Seminar 2012}

Diagnose und Therapie von Schwindelsyndromen, Okulomotorikund Gangstörungen

Am 6. und 7. Juli 2012 findet das sechzehnte Münchner Vertigo-Seminar unter der wissenschaftlichen Leitung von M. Strupp, M. Dieterich, E. Krause, K. Jahn und T. Brandt statt. Es wird gemeinsam vom Integrierten Forschungs- und Behandlungszentrum für Schwindel, Gleichgewichts-und Okulomotorikstörungen (IFB ${ }^{\mathrm{LMU}}$ ), der Neurologischen Klinik und der HNO-Klinik des Klinikums der Universität München veranstaltet.

Die Schwerpunkte dieser praxisorientierten Veranstaltung liegen am ersten Tag auf der Erhebung der Anamnese, der klinischen Untersuchung zur Differenzierung zwischen zentralen und peripheren Schwindelsyndromen, zerebellären Okulomotorikstörungen sowie der Differentialdiagnose des Phobischen Schwankschwindels, von Schwindelsyndromen bei Kindern und Gangstörungen. Am Ende des ersten Tages steht ein Videoquiz sowie eine offene Diskussion mit allen Referenten.
Am zweiten Tag werden parallel Kurse mit praktischen Übungen stattfinden. Hier liegt der Schwerpunkt auf okulomotorischen Störungen. Diese Kurse sind gleichermaßen geeignet für Neurologen, HNO- und Augenärzte, Orthoptistinnen und MTAs. Sie sollen in die körperlichen und apparativen Untersuchungstechniken sowie Therapieverfahren einführen und Kenntnisse sowie praktische Fertigkeiten vertiefen.

\section{Veranstalter:}

IFB ${ }^{\mathrm{LMU}}$, Neurologische Klinik und HNO-Klinik Klinikum der Universität München Campus Großhadern

Marchioninistraße 15, 81377 München

\section{Anmeldung:}

www.schwindelambulanz-muenchen.de

Anmeldeschluss ist der 15. Juni 2012 